\title{
Chromophore-assisted light inactivation of the V-ATPase V0c subunit inhibits neurotransmitter release downstream of synaptic vesicle acidification
}

Sylvain Rama ${ }^{1,2}$, Norah Boumedine-Guignon ${ }^{1,2}$, Marion Sangiardi ${ }^{1,2}$, Fahamoe Youssouf $^{1,2}$, Yves Maulet ${ }^{1,2}$, Christian Lévêque ${ }^{1,2}$, Maya Belghazi ${ }^{2,3}$, Michael Seagar ${ }^{1,2}$, Dominique Debanne ${ }^{1,2}$, Oussama EL FAR ${ }^{1,2}$

1. INSERM UMR_S 1072, 13015 Marseille, France

2. Aix-Marseille Université, 13015 Marseille, France

3. CNRS, UMR 7286, Plate-Forme de Recherche en Neurosciences PFRN, 13015, Marseille, France.

Correspondence and request for material should be addressed to O.E.F (email: oussama.el-far@inserm.fr; Tel: 00334916988 60; ORCID: 0000-0002-7473-8345)

\section{Keywords}

V-ATPase; Neurotransmission; Chromophore-assisted light inactivation (CALI); Hippocampal organotypic slice culture; Neurons; GeneGun.

\section{Abstract}

Synaptic vesicle proton V-ATPase is an essential component in synaptic vesicle function. Active acidification of synaptic vesicles, triggered by the V-ATPase, is necessary for neurotransmitter storage. Independently from its proton transport activity, an additional important function of the membrane-embedded sector of the VATPase has been uncovered over recent years. Subunits a and $\mathrm{c}$ of the membrane sector of this multi-molecular complex have been shown to interact with SNARE proteins and to be involved in modulating neurotransmitter release. The c-subunit interacts with the v-SNARE VAMP2 and facilitates neurotransmission. In this study, we used chromophore-assisted light inactivation and monitored the consequences on neurotransmission on line in CA3 pyramidal neurons. We show that V-ATPase csubunit VOc is a key element in modulating neurotransmission and that its specific inactivation rapidly inhibited neurotransmission.

\section{Keywords}

V-ATPase, Neurotransmission, Chromophore-assisted light inactivation (CALI), Hippocampal organotypic slice culture, Neurons, GeneGun 


\section{Acknowledgments}

We would like to thank S. J. Elledge for the generous gift of pINDUCER $10 \& 11$ and Nicolas Julien for advice on shRNA construction. The mass spectrometer was obtained using financial support from the "Fédération de Recherche pour le Cerveau" (FRC) through the Rotary operation "Espoir en tête". This work was supported by INSERM, the CNRS and the Agence Nationale pour la Recherche (grant ANR-11-BSV2-020MOMENT Blanc SVSE2.

\section{Introduction}

V-ATPase is an important effector in acidifying intracellular compartments in eukaryotes. This $\mathrm{pH}$ sensor [1] and mechano-chemical energy transducer [2] is composed of two reversibly attached intertwined multi-subunit domains V1 and V0 [3]. The multi-subunit $\mathrm{V} 1$ domain carries the ATPase activity and reversibly associates with the membrane-inserted proton transporter V0 domain. Although the structural organization of this enzyme is complex, its molecular integrity guarantees energydependent proton transfer. Regulated dissociation of its constituent domains modulates the coupling of ATP hydrolysis to proton transport and consequent acidification of subcellular compartments. V0 is composed of a hexamer of $16 \mathrm{kDa}$ hydrophobic V0c subunits with 4 transmembrane domains (TM) each, a $100 \mathrm{kDa}, 8$ TM V0a subunit, a soluble $40 \mathrm{kDa}$ V0d subunit and a $9 \mathrm{kDa}$ single TM V0e subunit, in addition to accessory proteins. Several lines of evidence now point to the involvement of V0 domain subunits; V0a [4,5] and V0c [6]; in regulating SNARE-mediated neurotransmitter release, independently of the ATPase and proton transport activity. In 2010, we showed that inhibiting V0c interaction with VAMP2 significantly decreases neurotransmission, highlighting the importance of VOc as a modulator of SNAREdependent neurotransmission [6]. Despite converging data on the implication of V0 subunits in neurotransmission, the mechanism by which the different V-ATPase V0 
subunits modulate neurotransmission is still obscure, especially for the extremely hydrophobic hexameric V0 c-subunit. Inactivation of the highly conserved V0 c-subunit [7] should allow further dissection of its role in neurotransmission. However the importance of this enzyme in cell viability through the acidification of different cellular compartments, complicates attempts at genetic inactivation [8-10] and in order to ascertain the role of c-subunit in neurotransmission, rapid and targeted protein inactivation techniques should represent an exploitable option.

Several membrane-permeant biarsenical dyes have been developed. They specifically bind an exogenous small tetracysteine (tc) motif genetically introduced into proteins of interest [11]. Upon intense illumination, these dyes produce short-lived (approximately $250 \mathrm{~ns}$ ) reactive oxygen species that locally and rapidly inactivate the tc-tagged protein $[11,12]$. This approach has already been successfully validated to study functional effects of protein inactivation $[13,11,14-16]$ including the implication of V0a1 subunit in neurotransmission. In the present study, we designed a light-sensitive V0c subunit and characterized the biochemical nature and consequences of protein damage. We then live-monitored synaptic transmission between connected CA3 pyramidal cells in organotypic slices following chromophore assisted light inactivation (CALI). Our results corroborate the implication of the V-ATPase V0c-subunit (ATP6V0c) in neurotransmitter release downstream of synaptic vesicle acidification.

\section{Material and Methods}

\section{Chemicals}

Spermidine, water-free ethanol and 2.3-dimercapto-1-propanol also known as British Anti-Lewisite (BAL) were from Sigma Aldrich. Bafilomycin A1, Tris(hydroxypropyl)phosphine (THP) and FiAsH-EDT2 were purchased from Santa 
Cruz Biotech. Early experimental setups were performed using FiAsH-EDT2 generously provided by Nicolas MOREL [5]. NiNTA agarose was from QIAGEN. Protease inhibitors (cOmplete) were from Roche Diagnostics GmbH. Anti-RFP antibodies were from Evrogen and doxycycline from Euromedex. Anti-HSV tag antibodies were from Abcam. HRP substrate TMB (3,3',5,5'-tetramethylbenzidine) was from Interchim. Neon electroporation apparatus (Thermofisher Scientific) was used for PC12 cell transfection. Helios GeneGun apparatus and chemicals for insert preparations were from BioRad. Fos- Choline-12 (n-dodecylphosphocholine) was from ANATRACE.

\section{ShRNA design}

The Public TRC (The RNAi Consortium) portal of the BROAD institute (https://portals.broadinstitute.org/gpp/public/) was used to predict a siRNA sequence that silences the expression of the RNA of the rat V0c subunit (ATP6V0c; NM_130823.3). siRNAV0c-391-412 5'-tgatcctgatcctcatctttg-3' was synthetized in a shRNA context (shRNAV0c-391-412:

TGCTGTTCAGACAGTGAGCGCtgatcctgatcctcatctttgTAGTGAAGCCACAGATGTAC agttaccaccacttgcacTIGCCTACTGCCTCGGA) where: the sequence in lowercase corresponds to the siRNA, underlined sequences to complementarity with subsequently used miR30 primers and the sequence in italics corresponds to the shRNA loop. The shRNA was amplified by PCR using "Phusion® High Fidelity Taq polymerase" with the following primers: the "miR30 context" forward Xho I (in italics) primer 5'-CAGAAGGCTCGAGAAGGTATATTGCTGTTGACAGTGAGCG-3' where the underlined sequence is complementary to the underlined 8 base pairs 5 ' to the 
siRNA sequence in the shRNA and the "miR30 context" reverse EcoR I (italics) primers 5'-CTAAAGTAGCCCCTTGAATTCCGAGGCAGTAGGCA-3' where the underlined sequence is complementary to the underlined 14 base pairs 3 ' to the shRNA.

\section{Cloning}

EGFP-ATP6V0c was prepared as described [6] using Sac I and Sal I restriction sites. The shRNA was inserted in pINDUCER10 [17] in the miR30 context using Xhol and EcoR I sites downstream of a doxycycline-inducible promotor (pINDUCER10-sh). In order to design a shRNA-insensitive V0c cDNA sequence, we used the degeneracy of the genetic code and changed the wobble base of degenerate codons throughout the native cDNA sequence (NM_130823.3) (Figure 3a). The wobbled cDNA sequence of ATP6V0c (wATP6V0c), encoding exactly the same amino acid sequence as the native protein, was synthesized by MWG Biofin and provided in $\mathrm{PEX}-\mathrm{E}$ vector. The shRNAinsensitive and tetracystein-tagged ATP6V0c (tcV0c) was constructed as follows: the sequence encoding the tetracystein tag (CCPGCC) was inserted, by directed mutagenesis, between isoleucine 4 and lysine 5 of wATP6V0c. The tcV0c was then amplified by PCR using Not I-flanked primers. Not I sites were introduced by mutagenesis in 5' and 3' of the EGFP sequence in pINDUCER11 and the subsequent Not I-EGFP-Not I sequence was excised and replaced by Not I-tcV0c-Not I sequence. Using directed mutagenesis, his-HSV-tagged tcV0c (HHtc-VOc) was constructed by adding the tetracystein tag between isoleucine 4 and lysine 5 of the previously described His-tagged HSV-V0c [6].

\section{Bacterial expression and purification of HHtc-V0c and GST-VAMP2}

C43 E.coli strain was used to express HHtc-V0c. Cultures were made in TB medium and the expression of the fusion protein was induced by $300 \mu \mathrm{M}$ IPTG over-night at 
$18^{\circ} \mathrm{C}$. Bacterial pellets were frozen at $-20^{\circ} \mathrm{C}$ until use. All the following steps were performed at $4{ }^{\circ} \mathrm{C}$. Nine grams of bacterial pellets were French pressed in resuspension buffer RP (20 ml Tris-HCl $10 \mathrm{mM} \mathrm{pH8,} \mathrm{EDTA} 1 \mathrm{mM}, 5 \mathrm{mM} \mathrm{L-methionine}$ containing cOmplete protease inhibitors and $1 \mathrm{mM}$ THP). The supernatant of a $5 \mathrm{~min}$ $2500 \times \mathrm{g}$ centrifugation was then centrifuged $30 \mathrm{~min}$ at $170000 \mathrm{xg}$ and the obtained pellet washed in RP using a Dounce homogenizer. Bacterial membranes were then resuspended at $10 \mathrm{mg} / \mathrm{ml}$ in Tris $50 \mathrm{mM} \mathrm{pH} 7.4, \mathrm{NaCl} 150 \mathrm{mM}$ and frozen at $-80^{\circ} \mathrm{C}$ until use. $15 \mathrm{ml}$ of bacterial membranes were used to solubilize HHtc-V0c by adding FosCholine12 (FC12) at $2 \%$ final concentration and protease inhibitors. The supernatant of a $170.000 \times \mathrm{g}$ centrifugation was then incubated for two hours with $1 \mathrm{ml}$ packed volume of Ni-NTA agarose in the presence of $20 \mathrm{mM}$ imidazole. After extensive washing with Tris $50 \mathrm{mM} \mathrm{pH} 7.4, \mathrm{NaCl} 1 \mathrm{M}, 50 \mathrm{mM}$ imidazole, $0.06 \% \mathrm{FC} 12$, the beads were rinsed with HEPES $50 \mathrm{mM} \mathrm{pH} 7.4, \mathrm{NaCl} 150 \mathrm{mM}, 0.06 \% \mathrm{FC} 12$ and eluted in the same buffer containing $0.5 \mathrm{M}$ imidazole. Aliquots of purified protein were stored at $80^{\circ} \mathrm{C}$ under nitrogen enriched atmosphere. GST-VAMP2 (2-92) and GST-VAMP2 (2-

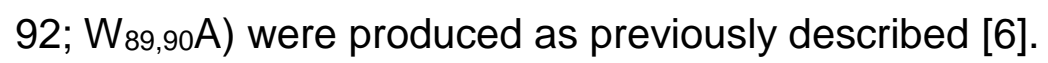

\section{In vitro chromophore assisted light inactivation}

$0.6 \mu \mathrm{g}(10 \mu \mathrm{l})$ of purified HHtc-V0c were deposited in the bottom of an opened Eppendorf tube in the absence or presence of $30 \mu \mathrm{M}$ FiAsh-EDT2. Tubes were placed on ice and subjected or not to 15 min direct illumination by a 100 watt light bulb positioned $1 \mathrm{~cm}$ above an open tube. Immediately after illumination, all tubes received $1 \mathrm{mM} \mathrm{BAL}$ and were vortexed. Samples were stored 6 hours at $4^{\circ} \mathrm{C}$ under nitrogenrich atmosphere before adding trypsin (200 $\mathrm{ng}$ ) and overnight digestion at $37^{\circ} \mathrm{C}$. Samples were then subjected to mass spectrometry with LTQ-Velos-Orbitrap (Thermo 
Fisher Scientific, Bremen, Germany) online analysis with a nanoliquid chromatography (LC) Ultimate 3000 system (Dionex, Sunnyvale, CA, USA). Parallel samples were used for ELISA VAMP2 binding tests or western blot analysis using anti HSV antibodies.

\section{Mass Spectrometry}

Samples were analyzed by mass spectrometry using a hybrid Q-Orbitrap mass spectrometer (Q-Exactive, Thermo Fisher Scientific, United States) coupled to a nanoliquid chromatography (LC) Dionex RSLC Ultimate 3000 system (Thermo Fisher Scientific, United States). Samples were trapped with a C18 PepMap 300 trap column $(300 \mu \mathrm{m} \times 5 \mathrm{~mm}, \mathrm{C} 18,5 \mu \mathrm{m}, 300 \AA$ ) and desalted with solvent $\mathrm{A}$ (water with $0.1 \%$ formic acid) for $3 \mathrm{~min}$ at a flow rate of $20 \mu \mathrm{L} / \mathrm{min}$. Peptide separation was performed on an Acclaim PepMap RSLC capillary column $(75 \mu \mathrm{m} \times 15 \mathrm{~cm}$, nanoViper C18, $2 \mu \mathrm{m}$, $100 \AA$ ) at a flow rate of $300 \mathrm{nl} / \mathrm{min}$. The analytical gradient was run with various percentages of solvent B (acetonitrile with $0.1 \%$ formic acid) in the following manner: (1) $2.5-25 \%$ for $57 \mathrm{~min}$, (2) $25-50 \%$ for $6 \mathrm{~min}$, (3) $50-90 \%$ for $1 \mathrm{~min}$, and (4) $90 \%$ for 10 min. MS spectra were acquired at a resolution of 35,000 within a mass range of $400-1,800 \mathrm{~m} / \mathrm{z}$. Ion accumulation was set at a maximum injection time of $100 \mathrm{~ms}$. Fragmentation spectra of the 10 most abundant peaks (Top10 method) were acquired with high-energy collision dissociation (HCD) at a normalized collision energy of $27 \%$. All raw data files generated by MS were processed to generate mgf files and searched against either the recombinant V0c sequence or the Uniprot E.coli database using the MASCOT software (www.matrixscience.com). Search parameters were as follow: variable modifications: oxidation of methionine, histidine, tryptophane, proline, cysteine, lysine and tyrosine residues; mass tolerance: $10 \mathrm{ppm}$ on parent ion and 0.02 Da on fragment ions, and a maximum of two tryptic missed cleavages. Peptide 
quantification was done using extracted ion chromatograms of peptide ions of interest in the software Xcalibur Qual Browser (Thermo Fisher Scientific, United States).

\section{ELISA}

Nunc Maxisorp 96-well plates were coated overnight with $100 \mu \mathrm{l}$ of CALI-treated or control HHtcV0c $(2 \mu \mathrm{g} / \mathrm{ml})$ in $100 \mathrm{mM} \mathrm{NaHCO}_{3}(\mathrm{pH}$ 8.9) buffer. After a $1 \mathrm{hr}$ blocking step with $25 \mathrm{mM}$ Tris-HCl (pH 7.5), $150 \mathrm{mM} \mathrm{NaCl}, 1 \%$ casein, $0.05 \%$ Tween 20, 300 nM of purified GST-VAMP2-(2-92) or GST-VAMP2-(2-92 $\mathrm{W}_{89,90 A}$ ) was added in $25 \mathrm{mM}$ Tris- $\mathrm{HCl}(\mathrm{pH} 7.5), 150 \mathrm{mM} \mathrm{NaCl}, 0.1 \% \mathrm{BSA}, 0.05 \%$ Tween 20 for $3 \mathrm{hrs}$ at $4^{\circ} \mathrm{C}$. Bound VAMP2 was then detected using the chromogenic HRP substrate TMB, consequent to the incubation with an anti-VAMP2 (mAb 6F9 [6]) (1:3000) first antibody and an HRPcoupled anti-mouse secondary antibody (Jackson ImmunoResearch).

\section{Organotypic cultures of rat hippocampus}

Hippocampal slice cultures were prepared as described previously [18]. Briefly, postnatal day 5-7 Wistar rats of both sexes were deeply anesthetized by intraperitoneal injection of chloral hydrate, the brain removed and each hippocampus individually dissected. Hippocampal slices $(350 \mu \mathrm{m})$ were placed on $20 \mathrm{~mm}$ latex membranes (Millicell) inserted into $35 \mathrm{~mm}$ Petri dishes containing $1 \mathrm{ml}$ of culture medium and maintained for up to 30 days in an incubator at $34^{\circ} \mathrm{C}, 95 \% \mathrm{O}_{2}-5 \% \mathrm{CO}_{2}$. The culture medium contained (in ml) 25 MEM, 12.5 HBSS, 12.5 horse serum, 0.5 penicillin $(10000 \mathrm{U} / \mathrm{ml}), 0.5$ streptomycin $(10 \mathrm{mg} / \mathrm{ml}), 0.8$ glucose $(1 \mathrm{M}), 0.1$ ascorbic acid $(1 \mathrm{mg} / \mathrm{ml}), 0.4$ HEPES ( $1 \mathrm{M}), 0.5 \mathrm{~B} 27,8.95$ sterile $\mathrm{H}_{2} \mathrm{O}$. To limit glial proliferation, $5 \mu \mathrm{M}$ Ara-C was added to the culture medium at 3 DIV for an overnight period.

\section{Transfection and Gene Gun}


PC12 cells were electroporated in solution following the manufacturer's instructions using the Neon ${ }^{\mathrm{TM}}$ transfection apparatus (1500 Volts, 3 pulses of $10 \mathrm{msec}$ each). Briefly, $3 \mu \mathrm{g}$ DNA (mixture of $1 \mu \mathrm{g}$ pEGFP-ATP6V0c and $2 \mu \mathrm{g}$ pINDUCER10-sh were used for $750 \times 10^{3}$ cells per well of a Nunc ${ }^{\mathrm{TM}} 24$ wells plate. Activation of the Tet-on tRFP and shRNA controlling promoter was achieved by adding $600 \mathrm{ng} / \mathrm{ml}$ of doxycycline to the culture medium 3 to 4 hours after transfection. Cells were recovered $48 \mathrm{~h}$ later, lysed and analysed by western blot using anti-GFP and anti-tRFP for ATP6V0c and shRNA expression levels respectively. Anti-GAPDH was used to normalize protein loading on gels. Relative EGFP-ATP6V0c expression after shRNA induction was quantified using Image J. Gene Gun bullets were prepared following manufacturer's instructions using $1 \mu \mathrm{m}$ gold micro-carriers and a gold $(\mathrm{mg}) / \mathrm{cDNA}(\mu \mathrm{g})$ ratio of 6/25. For hippocampal organotypic slice cultures, DIV 7-8 cultures were shot (1 $\mu \mathrm{g}$ DNA per bullet) at $120 \mathrm{psi}$. Doxycycline $(300 \mathrm{ng} / \mathrm{ml})$ was added one week after transfection and repeatedly supplemented every 36-48 hours until electrophysiological recordings between DIV 21 and 28 . Before recordings, cultures were perfused for 10 min with $1 \mu \mathrm{M}$ FiAsh-EDT 2 followed by a 20 min wash with $0.1 \mathrm{mM}$ BAL. In Bafilomycin conditions, after the baseline had stabilized, $50 \mu \mathrm{M}$ Bafilomycin were applied in the bath and neurons recorded for 40 minutes.

\section{Electrophysiological recordings and data analysis}

Dual whole-cell recordings were obtained from connected pairs of CA3 pyramidal neurons [18]. The external solution contained (mM): $125 \mathrm{NaCl}, 26 \mathrm{NaHCO}_{3}, 3 \mathrm{CaCl}_{2}$, $2.5 \mathrm{KCl}, 2 \mathrm{MgCl}_{2}, 0.8 \mathrm{NaH}_{2} \mathrm{PO}_{4}$ and $10 \mathrm{D}$-glucose, and was equilibrated with $95 \% \mathrm{O}_{2-}$ $5 \% \mathrm{CO}_{2}$. Patch pipettes (5-10 M $\Omega$ ) were filled with a solution containing (in $\mathrm{mM}$ ): 120 K-gluconate; $20 \mathrm{KCl} ; 10$ HEPES; 0.5 EGTA; $2 \mathrm{MgCl}_{2} ; 2$ Na $2 \mathrm{ATP} ; 0.3 \mathrm{NaGTP}$ (pH 7.4). All recordings were made at $30^{\circ} \mathrm{C}$ in a temperature-controlled recording chamber 
(Luigs \& Neumann, Ratingen, Germany). Both neurons were recorded with a Multiclamp 700B amplifier (Axon Instruments, Molecular Devices). The pre-synaptic cell was maintained in current clamp configuration and the post-synaptic cell in voltageclamp. Pre- and post-synaptic cells were held at their resting membrane potential ( $70 \mathrm{mV}$ ). Presynaptic APs were generated by injecting brief (10 ms) depolarizing pulses of current at a frequency of $0.1 \mathrm{~Hz}$. Paired-pulse ratio was assessed with two presynaptic stimulations delivered at a frequency of $20 \mathrm{~Hz}$. The voltage and current signals were low-pass filtered $(3 \mathrm{kHz})$, and acquisition of $1 \mathrm{~s}$ sequences was performed at $10 \mathrm{kHz}$ with pClamp (Axon Instruments) version 10. Data were analyzed with ClampFit (Axon Instruments) and custom made software written in LabView (National Instruments). Synaptic responses were averaged following alignment of the presynaptic action potentials using automatic peak detection. Pooled data are presented as mean \pm standard error to mean in all the figures, and statistical analysis was performed using the Mann-Whitney $U$-test or Wilcoxon rank-signed test with a significance level of $p<0.01$.

\section{Results}

Molecular consequences of tcV0c photo-damage. The reactive oxygen species generated by chromophore-assisted light inactivation (CALI) induce oxidization of susceptible residues nearby [19]. However, the biochemical consequences of protein photo-damage are not always addressed in CALI experiments. In order to address the extent of tcV0c damage after light exposure in the presence of the membranepermeant biarsenical dye FiAsh-EDT2 and apprehend the functional and molecular consequences of its potential modifications, we first analysed the oxidative state of the protein. Then, as we have previously shown that V0c interacts with VAMP2 to modulate 
neurotransmitter release [6], we addressed the consequences of photo-damage on this interaction. We used bacterially expressed $\mathrm{V} 0 \mathrm{c}(\mathrm{HHtcVOc})$ in which a tetra-cysteine tag was inserted in the $\mathrm{N}$-terminal domain (Figure 1a). The protein was purified under conditions that limit oxidization and subjected to an in vitro CALI protocol, in the absence or presence of FiAsh-EDT2 (See Materials and Methods). Samples were analysed by western blot and used in ELISA for VAMP2 binding assays. Their oxidative state was also analysed by mass spectrometry. Western blot analysis using an antiHSV antibody showed that under our experimental conditions, light exposure of the protein in the presence of FiAsh-EDT2 induced a slight shift in the migration profile of HHtcV0c (Figure 1b). The previously reported V0c dimer [6] also showed the same slight shift. No shift was observed using V0c devoid of tetra-cysteine tag and SDSresistant oligomeric tcV0c bands were not affected by the CALI protocol (Online Resource 1). Neither light exposure in the absence of FiAsh-EDT2, nor FiAsh-EDT2 addition in the absence of light, induced a similar shift of HHtcVOc (Figure 1). A very similar shift was previously observed upon investigating CALI-damaged calmodulin (CaM) [14]. Methionine oxidizations throughout the CaM protein sequence were found and the binding partner profile was different in pull-down assays using untreated or CALI-treated CaM [14]. We consequently analysed CALI-treated HHtcVOc by mass spectrometry and compared the oxidization state of light-exposed protein in the presence or absence of FiAsh-EDT2, and also FiAsh-EDT2 addition in the absence of light exposure. Tryptic digestion of HHtcV0c reproducibly generated four peptides (PEP1-4), illustrated in Online Resource 2a. PEP1 covered part of the N-terminal tag sequence upstream of the HSV tag, PEP2 covered TM 1 with an additional $10 \mathrm{~N}$ terminal amino acids, PEP3 covered loop 1-2 sequence and PEP4 covered principally TM3 (Online Resource 2a). All these peptides covered $45 \%$ of the V0c sequence. In 
all conditions, PEP4 did not show any oxidized residue. PEP1 and 2 showed significant oxidization on methionine residues, independently of CALI damage. PEP3 methionine residues M44, M47, M53 showed specific and extensive oxidation under light exposure in the presence of FiAsh-EDT2 (Online Resource 2b and Table 1). Analysis of the ratio of oxidized / un-oxidized peak surface areas of the different peptides showed that among all peptides, PEP 3 was the most affected. Triple oxidization of PEP3 dramatically increased (138 fold) after light exposure in the presence of FiAsh, in comparison to approximately 2 and 6 fold in the presence of light or FiAsh respectively. In order to address the specificity of oxidations, we analyzed, in parallel to HHtcVOc, the oxidation extent of the bacterial proteins SlyD that contaminates HHtcVOc preparations and co-purify on Ni-NTA-coupled beads. Tryptic digestion of SlyD generated five different peptides (not shown) covering $46 \%$ of the protein sequence. One of these peptides PepSlyD (Table 1) contained two methionine residues but did not show additional oxidation upon CALI treatment (Online Resource 2b). In order to investigate whether CALI treatment affected binding to VAMP2, we conducted ELISA experiments and tested the binding of GST-VAMP2 (1-92) to immobilized HHtcV0c. As shown in Figure 2, VAMP2 still binds to immobilized CALI-treated HHtcVOc very similar to non-modified or non-CALI treated V0c [6]. In both cases, this binding was dependent on the integrity of the two membrane proximal tryptophan residues $\left(\mathrm{W}_{89,90}\right)$ of VAMP2 that were shown to be important in binding to V0c [6]. These data indicate that CALI can generate oxidative damage to V0c in solution, without affecting interactions with VAMP2. Furthermore, in vivo, the diffusion of reactive oxygen species would be constrained by the synaptic vesicle membrane to intraluminal protein domains due to the $\mathrm{N}$-terminal position of the tetracysteine tag (see Discussion). 
Downregulation of V0c subunit by shRNA. In order to prepare and optimize the integration of light-sensitive VOc in functional protein complexes in living neurons, we reasoned that endogenous V0c levels should be partially downregulated. For this purpose we chose to use a shRNA approach. The unavailability of specific antibodies to follow modifications in endogenous V0c expression levels prompted us to evaluate the effectiveness of the shRNA approach in a heterologous system, where we measured the downregulation of exogenously expressed EGFP-V0c in the presence of anti-V0c shRNA. For this purpose, we co-transfected PC12 cells with two different plasmids, one expressing EGFP-V0c and the other anti-V0c shRNA. The latter was cloned in pINDUCER 10 in a miR30 context cassette, the expression of which is inducible by doxycycline. This miR30 backbone should promote long-term stability of the shRNA and therefore more efficient gene silencing [20]. EGFP-V0c expression levels were monitored using anti-EGFP antibodies and signals were normalized to GAPDH expression levels. Online Resource 3 shows that upon induction of anti-V0c shRNA expression versus the non-induced condition (Control), the EGFP signal was downregulated by $42.87 \% \pm 19.8 ; n=6$ independent experiments $p<0.01$ (MannWhitney U Test).

\section{Tetra-cysteine tagged ATP6-V0c insensitive to shRNA.}

As the proton pump activity of the V-ATPase is crucial for cell survival and in order to study the immediate effect of V0c inactivation on neurotransmission, we devised a means to replace, at least partially, endogeneous VOc by a light-sensitive version. CALI of Voc should allow its rapid inactivation and evaluate its implication in the final steps of neurotransmitter release. We therefore designed a plasmid construct (Figure 3a) that contained an inducible cassette expressing the fluorescent reporter protein tRFP and a shRNA that partially silences endogeneous V0c. The same plasmid 
constitutively expresses the reverse tet-transactivator rtTA3 and a tetra-cysteine tagged ATP6-V0c (tcV0c) at exactly the same position as in the bacterially expressed HHtcVOc. In order to limit protein modifications and allow the newly synthesised V0c to mix with endogenous non-modified V0c, this construct did not contain any additional tag (Figure 3a). The tcV0c DNA coding sequence was modified to render it insensitive to the shRNA (see material and methods). For this purpose, we used the degenerate property of the genetic code and exchanged every codon wobble base, keeping the original amino-acid sequence of V0c (Figure 3b). In the presence of doxycycline, rtTA3 binds and activates a tetracycline-responsive element upstream of the expression cassettes of tRFP and the anti-V0c shRNA in miR30 backbone coding sequences (Figure $3 \mathrm{a}, \mathrm{c}$ ).

Neuronal transfections and exogenous protein expression. Rat brain hippocampal organotypic cultures were transfected by GeneGun and the expression of tRFP as well as anti-VOc shRNA was induced by doxycycline. Transfected and induced cells were identified by tRFP fluorescence that homogeneously stained neuronal cell bodies and neurites (Figure 4a). Perfusion with the membrane permeable fluorescein-based arsenical dye FiAsh-EDT2 stained all cells. Excess and non-specific FiAsh-EDT2 binding was then extensively washed out in the presence of BAL, a potent FiAsh-EDT2 antidote that strongly binds to biarsenical dyes. Only tRFP-expressing cells kept fluorescein staining, confirming the specific retention of FiAsh-EDT2 by neurons expressing tcV0c (Figure 4b).

\section{Photo-inactivation of tcV0c inhibits neurotransmission between connected CA3}

neurons. Synaptic transmission was continuously monitored in connected CA3 neuronal pairs throughout the photo-inactivation protocol. tRFP-positive neurons were patched and maintained in current clamp (Figure 5a). Neighbouring synaptically- 
connected neurons were identified and recorded in voltage-clamp configuration. Presynaptic action potentials were continuously elicited at $0.1 \mathrm{~Hz}$ by injecting $10 \mathrm{~ms}$ depolarizing current pulses. When the baseline had stabilized (around $10 \mathrm{~min}$ ), a 100 watt illumination was applied for $5 \mathrm{~min}$. As shown in Figure $5 \mathrm{~b}$ bottom and $5 \mathrm{c}$ right, illumination did not significantly affect baseline recordings in non-transfected neurons (3\% $\% 8 \% ; n=8 ; p>0.1)$. In contrast, a significant decrease $(33 \% \pm 4 \% ; n=8 ; p<0.01)$ was observed in postsynaptic responses of neurons connected to tcV0c-expressing presynaptic ones (Figure 5b Top, 5c left, 5d). The time course of CALI versus Bafilomycin induced inhibition of neurotransmission displayed respectively $\tau=2.04$ and 10.4 min (Online Resource 4) suggesting that CALI induced inhibition is not related to proton pump inhibition. Paired pulse ratio measurement in CALI-treated neurons showed no significant change $(66 \pm 4$ versus $62 \pm 6 \% n=8$, Wilcoxon $p=0.67$ before and after light exposure respectively). No change in presynaptic input resistance and capacitance was observed in the CALI-treated neurons (respectively, $96 \pm 4 \%$ of the control resistance, Wilcoxon test, $\mathrm{p}>0.1$ and $96 \pm 4 \%$ of the control capacitance). This indicates that the free-radicals produced by CALI treatment did not affect the passive properties of the presynaptic neuron. It is noticeable that illuminating tRFP and tcV0c expressing cells in the absence of FiAsh-EDT2 resulted in moderate, but nonsignificant decrease in postsynaptic responses $(16 \% \pm 5 \% ; n=8 ; p>0.01$ ) (Figure $5 d, e)$, presumably due to tRFP photo-damage. Post-synaptic input resistance was unchanged in all cases (following CALI-treatment: $93 \pm 3 \%$, Wilcoxon test $p>0.01$; in non-transfected cells: $99 \pm 3 \%$, Wilcoxon test $p>0.1$ and in transfected cells without FiAsh: $97 \pm 5 \%$, Wilcoxon test $p>0.1$ ). No change in post-synaptic membrane capacitance was observed in all cases (from $58 \pm 6 \mathrm{pF}$ to $57 \pm 5 \mathrm{pF}$ for CALI-treated 
neurons, $63 \pm 12 \mathrm{pF}$ to $59 \pm 12 \mathrm{pF}$ for untransfected neurons and from $84 \pm 9 \mathrm{pF}$ to 89 $\pm 11 \mathrm{pF}$ for transfected neurons recorded in the absence of FiAsh.

\section{Discussion}

The V-ATPase is thus thought to play two roles in neurotransmitter release at different stages in the synaptic vesicle cycle, (i) establishing the proton gradient that transporters use to load vesicles with transmitter; (ii) modulate synaptic vesicle fusion at the plasma membrane. In this second role, more than three decades ago, experiments designed to identify proteins involved in acetylcholine release from Torpedo synaptosomes, uncovered a non-elucidated role of V0c subunit in quantal and calcium-dependent acetylcholine release [21-24]. More than a decade ago, studies on yeast vacuolar fusion mechanisms uncovered a crucial role of $\mathrm{V} 0 \mathrm{c}$ and a-subunits in membrane fusion downstream of SNARE complex assembly $[25,26]$. Subsequently, an unbiased genetic screen for synaptic malfunction in Drosophila identified the a1subunit of $\mathrm{V} 0$ as an essential component in evoked synaptic transmission, also acting downstream of SNAREs [27]. Interestingly, it was also shown that $\mathrm{Ca}^{2+/ c a l m o d u l i n}$ interaction with V0a specifically controlled spontaneous neurotransmitter release at Drosophila neuromuscular junction [28]. Moreover, independently from its $\mathrm{H}^{+}$transport function, it has been shown that the a-subunit of the V-ATPase regulates insulin secretion in pancreatic beta-cells [29], lysosome-phagosome fusion in zebrafish [30] and plays a crucial role in exosome-mediated apical secretion of Hedgehog-related proteins in C.elegans [31]. The fact that alternative splicing controls differential sorting of V0 a1-subunit variants to nerve terminals or distal dendrites is also consistent with a specific role for a-subunits in neurosecretion [32]. In mice, inhibiting V0c interaction with VAMP2 significantly decreases neurotransmission, highlighting the importance of V0c as a modulator of higher vertebrate's SNARE-dependent neurotransmission [6]. 
Molecularly, the V0 domain subunits of the V-ATPase have been shown to coimmunoprecipitate with SNARE proteins, the calcium sensor synaptototagmin, as well as the synaptic vesicle proteins synaptotaphysin, and SV2 [33,34]. Chromophoreassisted light inactivation is a very useful technology that allows live-monitoring of the functional consequences of rapid and targeted protein inactivation [19]. In this report, we describe targeted photo-inactivation of the V-ATPase V0c subunit, analyse its biochemical modifications and monitor the consequences on neurotransmission of its inactivation in living neurons. In synaptic vesicle membranes, V0c is very tightly compacted in the membrane and only loops 1-2 and 3-4 are exposed to the cytosolic side. The $\mathrm{N}$ and $\mathrm{C}$-termini as well as loop 2-3 are intraluminal. In order to examine the biochemical modifications that light inactivation can induce in V0c, we used bacteriallyexpressed protein (HHtcV0c) in which a tetra-cysteine tag was inserted in the nonconserved N-terminal part. The choice of the site of tag insertion was driven by the very compact structure of the protein and that modification in the loops might perturb protein structure and complicate functional conclusions. Western blot analysis of in vitro photo-inactivated V0c showed a slight shift in the apparent molecular weight of the protein. This shift resembled the electrophoretic behaviour of oxidized proteins as described for S100A9 [35] and calmodulin after CALI treatment [14]. In order to identify potential oxidization hot spots in CALI-treated VOc, we analysed by mass spectrometry the oxidation profile of amino acids susceptible to oxidization [19] (tryptophan, tyrosine, histidine, cysteine, proline, lysine and methionine). We addressed the specificity of the damage induced by oxygen radicals, by analysing the extent of SlyD oxidation, a bacterial contaminant chaperone that co-purified with HHtcVOc. Mass spectrometry analysis of tryptic peptides generated by CALI in solution indicated that, in all conditions, no significant difference was observed in oxidation levels of SlyD peptide. 
Among all amino acid residues analysed, only methionines were oxidized and most of the photo-damage was found in the cytoplasmic V0c loop 1-2. It is noteworthy however that biochemical damage observed in vitro may be unrelated to that which occurs in a living cell. In vivo singlet oxygen should only have access to protein moieties located inside synaptic vesicles: the $\mathrm{N}$-terminus of the protein, loop 2-3 and potentially parts of TM1 that was shown to be accessible to hydrophilic compounds in arthropods [36]. Oxidization might also affect V0c oligomerization status and potentially its interaction with other molecular partners. By introducing a tetracysteine tag in the $\mathrm{N}$-terminal extravesicular side of the V-ATPase V0a subunit, an earlier report [37] has addressed the consequence of damaging this subunit on neurotransmission. Although careful control recordings were performed, especially to verify that damaging subunits of the V1 domain did not inhibit neurotransmission, extra-vesicular generation of singlet oxygens could very locally damage surrounding cytosolic effectors unrelated to VATPase and implicated in SNARE-dependent fusion. In the configuration we used, the synaptic vesicle membrane should serve as a shield and protect vicinal cytosolic proteins, as well as the cytosolic moiety of synaptic vesicle membrane proteins from oxygen radicals.

For experiments in living cells, we combined in the same vector the constitutive expression of a tetracysteine tagged V0c subunit and an inducible cassette coupling the expression of tRFP as a fluorescent transfection marker and an shRNA that specifically downregulates endogenous V0c subunit. Specific chromophore-assisted light inactivation of V0c subunit in a presynaptic neuron rapidly (2-3 min) inhibited neurotransmission between connected CA3 pyramidal pairs. Interestingly, our ELISA experiments showed that the CALI treated V0c still interacts with VAMP2 with the same specificity criterion as wild type V0c. These results suggested that the observed 
decrease in neurotransmission upon CALI in living neurons is not due to a change in V0c binding to VAMP2. This is corroborated by the fact that no change in PPR was observed after CALI, while perturbing VAMP2 / V0c interaction lead to a significant increase in PPR [6]. Monitoring neuronal activity in connected non-transfected pairs guaranteed that the stimulation frequency was sufficiently moderate to exclude ready releasable pool depletion. In addition, in reciprocally connected pairs, the CALI protocol only reduced neurotransmission when the presynaptic neuron was transfected (not shown).

Exogenous overexpression of tcV0c might increase the plasma membrane pool of $\mathrm{V}$ ATPase all over the neuronal plasma membrane and hence facilitate endocytosis through alkalinisation of the cytosol [38]. Our data from reciprocally connected neurons argue against this interpretation since monitoring neurotransmission from a nontransfected presynaptic neuron towards a CALI treated postsynaptic neuron and vice versa do not show any sign of membrane capacitance changes. It could also be argued that singlet oxygens can damage the membranes in which the protein is inserted and therefore lead to changes in membrane permeability, inducing leakage either in neuronal plasma membranes or synaptic vesicles. Our electrophysiological recordings also argue against this interpretation since membrane resistance changes were not statistically significant. In our recording conditions, any impact on vesicular acidification should also be negligible since the stimulation frequency is far too low to deplete reserve synaptic vesicle pools and high concentrations of Bafilomycin, which block the proton pump, showed 5 times slower inhibition kinetics than CALI treated samples.

In conclusion, the present study confirms and strengthens our former findings about the important role of the V-ATPase VOc membrane sector in modulating neurotransmitter release [6]. It suggests that CALI-triggered V0c inactivation induces 
distinct molecular perturbations that do not affect interaction with VAMP2 and that V0c is positioned strategically close to the site of exocytosis.

Fig. 1

Electrophoretic behavior of CALI-treated VOc. a Amino acid sequence of HHtcVOc; transmembrane sequences are boxed; tryptic peptides identified in mass spectrometry are highlighted in bold. Tetracysteine tag is underlined. N-terminal tags are in italics $\mathbf{b}$ Lane 1 shows coomassie staining of purified HHtcV0c; $\left({ }^{*}\right)$ monomer and $\left({ }^{* *}\right)$ dimer with respective apparent molecular weights of 18 and $38 \mathrm{kDa}$. Purified HHtcVOc was subjected to illumination (lanes 4 and 5) in the presence (lanes 3 and 5) or absence (lanes 2 and 4 ) of $30 \mu \mathrm{M}$ FiAsh and analyzed by western blot using anti-HSV tag antibodies. Like HHtcV0c [6], anti-HSV antibodies detected SDS-resistant multimers of V0c. A small increase of approximately $2 \mathrm{kDa}$ was observed in the apparent molecular weight of monomers and dimers of CALI damaged HHtcVO (No: nonoxidized and Ox: oxidized). Results are representative of more than 3 independent experiments.

\section{Fig. 2}

VAMP2 binding to CALI treated HHtcVOc. CALI-treated HHtcVOc was immobilized and binding of wild type and mutant ( $\mathrm{W}_{89,90 A}$ ) GST-VAMP2 was assessed by ELISA using anti GST antibodies. Results are representative of 3 independent experiments.

\section{Fig. 3}


tcV0c expression construct. a Diagram of relevant cassettes in V0c-expressing pINDUCER-11; tRFP: turbo Red Fluorescent protein; miR30-shV0c: anti-V0c shRNA; rtTA3: reverse tet-transactivator, IRES: Internal ribosome entry site; tcV0c: tetracysteine containing, sh-resistant V0c b cDNA sequence of shRNA-insensitive V0c; nucleotides indicated in red correspond to the substituted third nucleotide of codons. Boxed sequence is the shRNA targeted sequence on V0c. c Secondary structure representation of anti-V0c shRNA in miR30 backbone (RNAfold WebServer; http://rna.tbi.univie.ac.at)

\section{Fig. 4}

Fluorescent detection of tcV0c expressing cells. a Representative example of a proximal pyramidal CA3 neuron in an organotypic hippocampal rat brain culture transfected by GeneGun using tcV0c expression vector and expressing tRFP after doxycycline induction. b. FiAsh fluorescence of the tRFP-expressing cell in a confirming the expression of tcV0c. Scale bars: $50 \mu \mathrm{m}$

\section{Fig. 5}

Effect of V0c photoinactivation on synaptic transmission in CA3 pyramidal neurons of cultured hippocampal slices. a A tRFP fluorescent capture of a typical experiment showing pair-recording of CA3 pyramidal cells. Pipette 1 is recording a tcV0c transfected neuron (1), while pipette 2 records a non-transfected postsynaptic neuron (2) (delimited with a dashed line) connected to neuron 1. Scale Bar: $50 \mu \mathrm{m}$. b Example of paired recordings: action potentials are induced in current clamp in presynaptic cell 1 and EPSCs are recorded in postsynaptic cell 2. Top: The presynaptic neuron 1 expresses tcV0c (example shown in a). Bottom: both cells are non-transfected. c Illustration of an example of EPSC amplitudes evolution before and after light exposure 
in single tcVOc (left, red) and control neurons (right, black). Note the decrease of EPSC amplitude when the presynaptic cell expresses tcV0c. Arrows indicate example traces shown in b. d Time course of relative synaptic response amplitudes when presynaptic cells express tcV0c and are subjected to full CALI protocol (upper), presynaptic cells are non-transfected and full CALI protocol applied (middle) and when presynaptic cells express tcV0c and are subjected to full CALI protocol in the absence of FiAsh (lower). The grey and dotted areas represent respectively the illumination period and areas used for statistical analysis. Amplitudes are mean recordings from 8 different neurons and error bars represent the standard error of the mean (SEM) e Relative comparison between all experimental conditions. Open circles show individual experiments. Note the slight decrease of postsynaptic responses in the absence of FiAsh-EDT2 (blue) is probably due to oxygen species released after photodamage of tRFP

\section{Table 1}

Mass spectrometric identification of tryptic peptides

\section{Notes}

\section{Compliance with ethical standards}

This study does not involve experiments on live animals. All experiments were carried out according to the European and Institutional guidelines for the care and use of laboratory animals (Council Directive 86/609/EEC and French National Research Council) and were approved by the local health authority (\# D 13055 08, Préfecture des Bouches-du-Rhône, Marseille).

\section{Conflict of Interest}


The authors declare that they have no conflict of interest.

\section{References}

1. Hurtado-Lorenzo A, Skinner M, El Annan J, Futai M, Sun-Wada GH, Bourgoin S, Casanova J, Wildeman A, Bechoua S, Ausiello DA, Brown D, Marshansky V (2006) V-ATPase interacts with ARNO and Arf6 in early endosomes and regulates the protein degradative pathway. Nature cell biology 8 (2):124-136

2. Yokoyama K, Nakano M, Imamura H, Yoshida M, Tamakoshi M (2003) Rotation of the proteolipid ring in the V-ATPase. J Biol Chem 278 (27):24255-24258

3. Toei M, Saum R, Forgac M (2010) Regulation and Isoform Function of the V-ATPases. Biochemistry 49 (23):4715-4723

4. Hiesinger PR, Fayyazuddin A, Mehta SQ, Rosenmund T, Schulze KL, Zhai RG, Verstreken P, Cao Y, Zhou Y, Kunz J, Bellen HJ (2005) The v-ATPase V(0) Subunit a1 Is Required for a Late Step in Synaptic Vesicle Exocytosis in Drosophila. Cell 121 (4):607-620

5. Poea-Guyon S, Ammar MR, Erard M, Amar M, Moreau AW, Fossier P, Gleize V, Vitale N, Morel N (2013) The V-ATPase membrane domain is a sensor of granular $\mathrm{pH}$ that controls the exocytotic machinery. J Cell Biol 203 (2):283-298

6. Di Giovanni J, Boudkkazi S, Mochida S, Bialowas A, Samari N, Leveque C, Youssouf F, Brechet A, Iborra C, Maulet Y, Moutot N, Debanne D, Seagar M, El Far O (2010) V-ATPase membrane sector associates with synaptobrevin to modulate neurotransmitter release. Neuron 67 (2):268-279

7. El Far O, Seagar M (2011) A role for V-ATPase subunits in synaptic vesicle fusion? J Neurochem 117 (4):603-612. doi:10.1111/j.1471-4159.2011.07234.x

8. Hayami K, Noumi T, Inoue H, Sun-Wada G, Yoshimizu T, Kanazawa H (2001) The murine genome contains one functional gene and two pseudogenes coding for the $16 \mathrm{kDa}$ proteolipid subunit of vacuolar H(+)-ATPase. Gene 273 (2):199-206

9. Sun-Wada G, Murata Y, Yamamoto A, Kanazawa H, Wada Y, Futai M (2000) Acidic endomembrane organelles are required for mouse postimplantation development. Dev Biol 228 (2):315-325. doi:10.1006/dbio.2000.9963

10. Inoue H, Noumi T, Nagata M, Murakami H, Kanazawa H (1999) Targeted disruption of the gene encoding the proteolipid subunit of mouse vacuolar $\mathrm{H}(+)$-ATPase leads to early embryonic lethality. Biochim Biophys Acta 1413 (3):130-138

11. Tour O, Meijer RM, Zacharias DA, Adams SR, Tsien RY (2003) Genetically targeted chromophoreassisted light inactivation. Nat Biotechnol 21 (12):1505-1508. doi:10.1038/nbt914

12. Ochsner M (1997) Photophysical and photobiological processes in the photodynamic therapy of tumours. J Photochem Photobiol B 39 (1):1-18

13. Sano Y, Watanabe W, Matsunaga S (2014) Chromophore-assisted laser inactivation - towards a spatiotemporal-functional analysis of proteins, and the ablation of chromatin, organelle and cell function. J Cell Sci 127 (8):1621-1629. doi:10.1242/jcs.144527

14. Yan P, Xiong Y, Chen B, Negash S, Squier TC, Mayer MU (2006) Fluorophore-assisted light inactivation of calmodulin involves singlet-oxygen mediated cross-linking and methionine oxidation. Biochemistry 45 (15):4736-4748. doi:10.1021/bi052395a

15. Marek KW, Davis GW (2002) Transgenically encoded protein photoinactivation (FIAsH-FALI): acute inactivation of synaptotagmin I. Neuron 36 (5):805-813

16. Morel N, Poea-Guyon S, Ammar MR, Vitale N (2014) [V-ATPase is a pH sensor controlling vesicular membrane fusion]. Med Sci (Paris) 30 (6-7):631-633. doi:10.1051/medsci/20143006011 
17. Meerbrey KL, Hu G, Kessler JD, Roarty K, Li MZ, Fang JE, Herschkowitz JI, Burrows AE, Ciccia A, Sun T, Schmitt EM, Bernardi RJ, Fu X, Bland CS, Cooper TA, Schiff R, Rosen JM, Westbrook TF, Elledge SJ The pINDUCER lentiviral toolkit for inducible RNA interference in vitro and in vivo. Proceedings of the National Academy of Sciences of the United States of America 108 (9):3665-3670

18. Debanne D, Boudkkazi S, Campanac E, Cudmore RH, Giraud P, Fronzaroli-Molinieres L, Carlier E, Caillard O (2008) Paired-recordings from synaptically coupled cortical and hippocampal neurons in acute and cultured brain slices. Nat Protoc 3 (10):1559-1568. doi:10.1038/nprot.2008.147

19. Lin JY, Sann SB, Zhou K, Nabavi S, Proulx CD, Malinow R, Jin Y, Tsien RY (2013) Optogenetic inhibition of synaptic release with chromophore-assisted light inactivation (CALI). Neuron 79 (2):241-253. doi:10.1016/j.neuron.2013.05.022

20. Meerbrey KL, Hu G, Kessler JD, Roarty K, Li MZ, Fang JE, Herschkowitz JI, Burrows AE, Ciccia A, Sun T, Schmitt EM, Bernardi RJ, Fu X, Bland CS, Cooper TA, Schiff R, Rosen JM, Westbrook TF, Elledge SJ (2011) The pINDUCER lentiviral toolkit for inducible RNA interference in vitro and in vivo. Proc Natl Acad Sci U S A 108 (9):3665-3670. doi:10.1073/pnas.1019736108

21. Israel M, Morel N, Lesbats B, Birman S, Manaranche R (1986) Purification of a presynaptic membrane protein that mediates a calcium-dependent translocation of acetylcholine. Proc Natl Acad Sci U S A 83 (23):9226-9230

22. Falk-Vairant J, Meunier FM, Lesbats B, Correges P, Eder-Colli L, Salem N, Synguelakis M, Dunant $Y$, Israel M (1996) Cell lines expressing an acetylcholine release mechanism; correction of a releasedeficient cell by mediatophore transfection. J Neurosci Res 45 (3):195-201. doi:10.1002/(SICI)10974547(19960801)45:3\&lt;195::AID-JNR1\&gt;3.0.CO;2-8

23. Falk-Vairant J, Correges P, Eder-Colli L, Salem N, Roulet E, Bloc A, Meunier F, Lesbats B, Loctin F, Synguelakis M, Israel M, Dunant Y (1996) Quantal acetylcholine release induced by mediatophore transfection. Proc Natl Acad Sci U S A 93 (11):5203-5207

24. Falk-Vairant J, Correges P, Eder-Colli L, Salem N, Meunier FM, Lesbats B, Loctin F, Synguelakis M, Israel M, Dunant $Y(1996)$ Evoked acetylcholine release expressed in neuroblastoma cells by transfection of mediatophore cDNA. J Neurochem 66 (3):1322-1325

25. Peters C, Bayer MJ, Buhler S, Andersen JS, Mann M, Mayer A (2001) Trans-complex formation by proteolipid channels in the terminal phase of membrane fusion. Nature 409 (6820):581-588. doi:10.1038/35054500

26. Bayer MJ, Reese C, Buhler S, Peters C, Mayer A (2003) Vacuole membrane fusion: V0 functions after trans-SNARE pairing and is coupled to the Ca2+-releasing channel. J Cell Biol 162 (2):211-222. doi:10.1083/jcb.200212004

27. Hiesinger PR, Fayyazuddin A, Mehta SQ, Rosenmund T, Schulze KL, Zhai RG, Verstreken P, Cao Y, Zhou Y, Kunz J, Bellen HJ (2005) The v-ATPase V0 subunit a1 is required for a late step in synaptic vesicle exocytosis in Drosophila. Cell 121 (4):607-620. doi:10.1016/j.cell.2005.03.012

28. Wang D, Epstein D, Khalaf O, Srinivasan S, Williamson WR, Fayyazuddin A, Quiocho FA, Hiesinger PR (2014) Ca2+-Calmodulin regulates SNARE assembly and spontaneous neurotransmitter release via V-ATPase subunit V0a1. J Cell Biol 205 (1):21-31. doi:10.1083/jcb.201312109

29. Sun-Wada GH, Toyomura T, Murata Y, Yamamoto A, Futai M, Wada Y (2006) The a3 isoform of VATPase regulates insulin secretion from pancreatic beta-cells. J Cell Sci 119 (Pt 21):4531-4540. doi:10.1242/jcs.03234

30. Peri F, Nusslein-Volhard C (2008) Live imaging of neuronal degradation by microglia reveals a role for v0-ATPase a1 in phagosomal fusion in vivo. Cell 133 (5):916-927. doi:10.1016/j.cell.2008.04.037

31. Liegeois S, Benedetto A, Garnier JM, Schwab Y, Labouesse M (2006) The V0-ATPase mediates apical secretion of exosomes containing Hedgehog-related proteins in Caenorhabditis elegans. J Cell Biol 173 (6):949-961. doi:10.1083/jcb.200511072

32. Poea-Guyon S, Amar M, Fossier P, Morel N (2006) Alternative splicing controls neuronal expression of v-ATPase subunit a1 and sorting to nerve terminals. J Biol Chem 281 (25):17164-17172. doi:10.1074/jbc.M600927200

33. Baldwin MR, Barbieri JT (2007) Association of botulinum neurotoxin serotypes a and B with synaptic vesicle protein complexes. Biochemistry 46 (11):3200-3210. doi:10.1021/bi602396x 
34. Galli T, McPherson PS, De Camilli P (1996) The Vo sector of the V-ATPase, synaptobrevin, and synaptophysin are associated on synaptic vesicles in a Triton X-100-resistant, freeze-thawing sensitive, complex. J Biol Chem 271 (4):2193-2198

35. Sroussi HY, Berline J, Palefsky JM (2007) Oxidation of methionine 63 and 83 regulates the effect of S100A9 on the migration of neutrophils in vitro. J Leukoc Biol 81 (3):818-824. doi:10.1189/jlb.0706433 36. Jones PC, Harrison MA, Kim YI, Finbow ME, Findlay JBC (1995) The first putative transmembrane helix of the $16 \mathrm{kDa}$ proteolipid lines a pore in the $\mathrm{V}<\mathrm{sub}>0</ \mathrm{sub}>$ sector of the vacuolar $\mathrm{H}<$ sup $>+<$ /sup $>$-ATPase. Biochemical Journal 312 (3):739-747. doi:10.1042/bj3120739

37. Poea-Guyon S, Ammar MR, Erard M, Amar M, Moreau AW, Fossier P, Gleize V, Vitale N, Morel N (2013) The V-ATPase membrane domain is a sensor of granular $\mathrm{pH}$ that controls the exocytotic machinery. J Cell Biol 203 (2):283-298. doi:10.1083/jcb.201303104

38. Zhang Z, Nguyen KT, Barrett EF, David G Vesicular ATPase Inserted into the Plasma Membrane of Motor Terminals by Exocytosis Alkalinizes Cytosolic pH and Facilitates Endocytosis. Neuron 68 (6):1097-1108. doi:10.1016/j.neuron.2010.11.035 


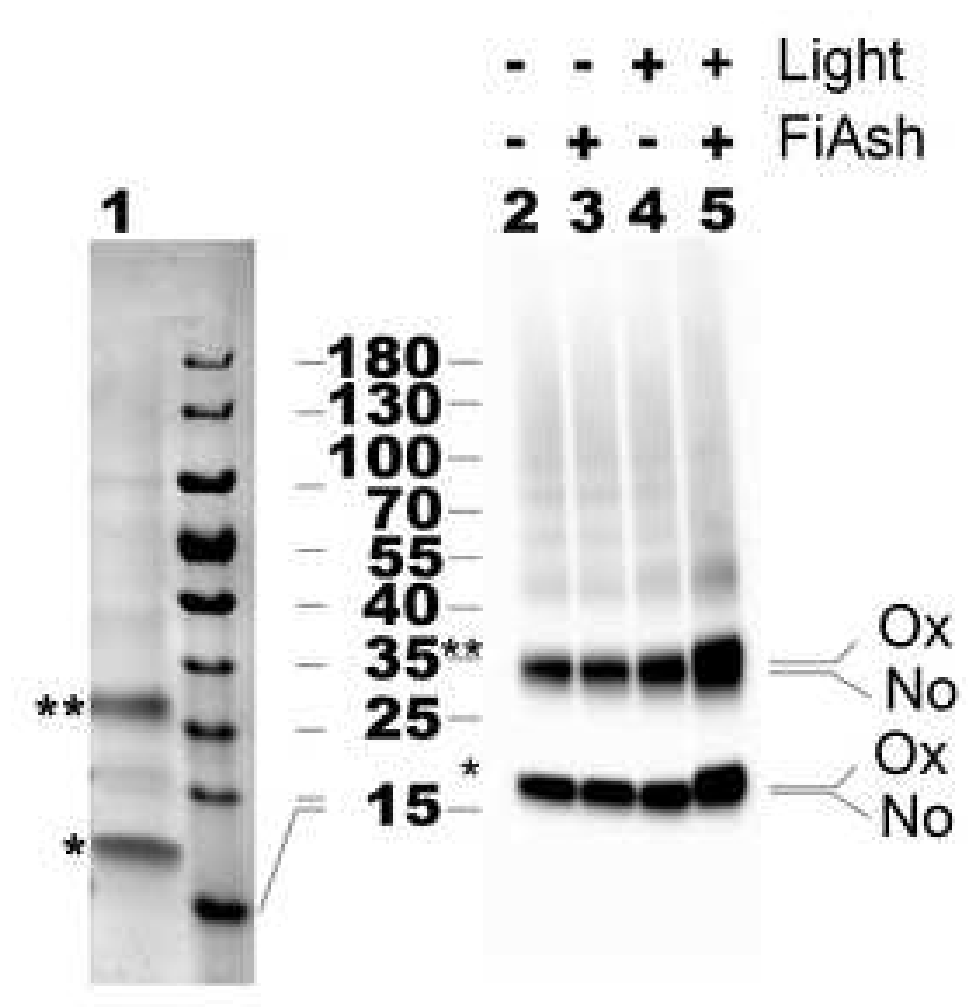




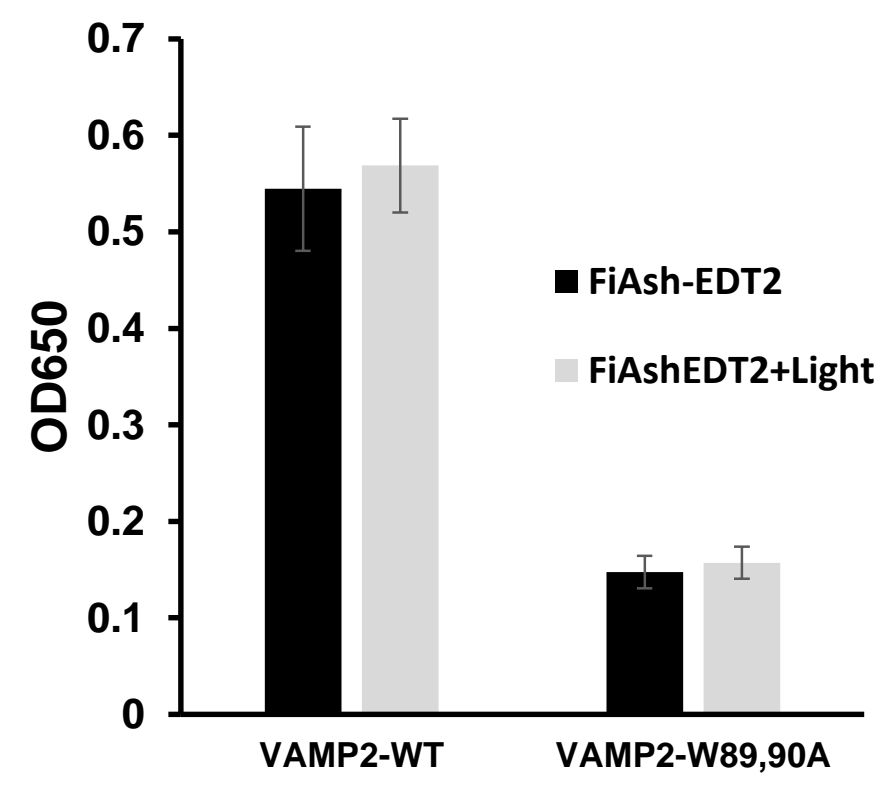

Figure 2 
a

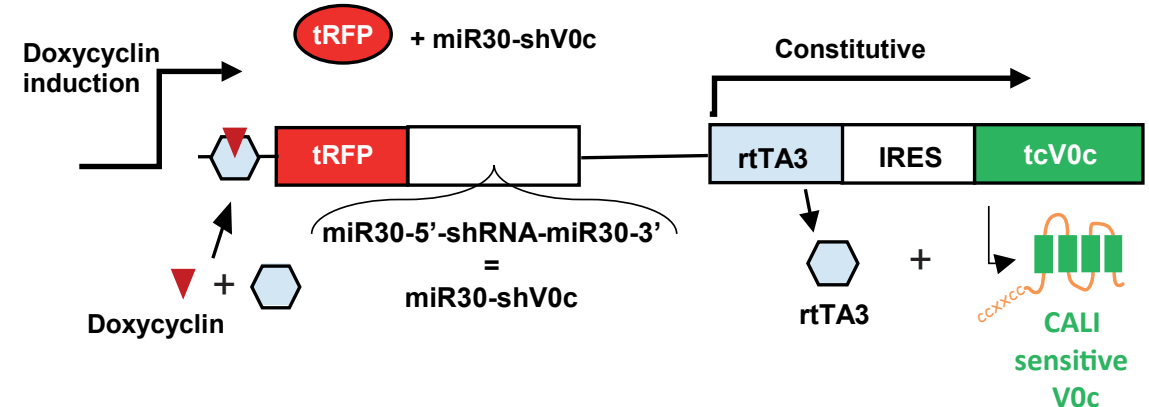

b

ATGGCTGACATCAAGAACAACCCCGAATATTCTTCGTTTTTCGGTGTCATGGGCGCCTCGTCCGCCATGGTCTTC $\begin{array}{llllllllllllllllllllll}G & T & T & A & T & T & A & G & C & G & A & C & T & C & A & \text { T } & \text { A } & \text { T } & \text { A } & \text { T } & \text { A } & \text { T }\end{array}$ AGCGCCATGGGAGCTGCCTATGGCACAGCCAAGAGTGGCACTGGCATCGCAGCCATGTCGGTCATGAGGCCAGAG $\begin{array}{lllllllllllllllllllllllllll}T & T & G & C & T & C & T & C & T & A & C & T & G & A & A & G & T & & T & A & A & G & A\end{array}$ CTGATCATGAAGTCCATCATCCCAGTGGTTATGGCTGGGATCATCGCCATCTACGGCCTGGTGGTTGCAGTACTT $\begin{array}{llllllllllllllllllllllllll}\text { A } & \text { A } & \text { A } & \text { T } & \text { A } & \text { T } & G & \text { T } & \text { C } & & \text { C } & \text { A } & \text { T } & \text { A } & \text { A } & \text { T } & \text { T } & \text { G } & \text { C } & \text { T } & \text { C } & G & \text { T } & \text { G }\end{array}$ ATCGCTAACTCCCTGACTGATGGCATCACCCTCTACAGGAGTTTTCTTCAACTGGGTGCTGGCCTGAGTGTGGGG $\begin{array}{lllllllllllllllllllllllllll}A & C & T & T & C & G & C & G & T & G & G & T & A & C & C & G & G & C & A & A & T & C & C & A & C\end{array}$ CTGAGTGGCCTGGCTGCTGGCTTTGCCATTGGCATTGTCGGAGATGCTGGTGTCCGGGGCACTGCCCAGCAGCCT

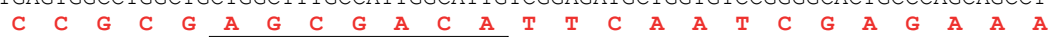
CGACTGTTCGTGGGCATGATCCTGATCCTCATCTTTGCGGAGGTGCTTGGCCTCTACGGTCTCATCGTGGCCCTA

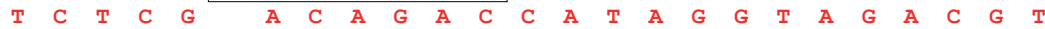
ATCCTCTCCACAAAGTAG

A $G \quad G \quad T$ A $A$

C

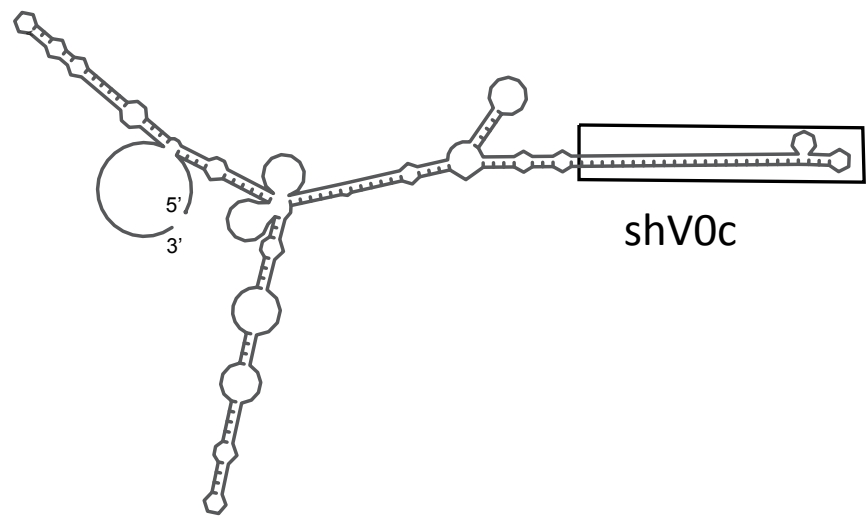


Figure 4

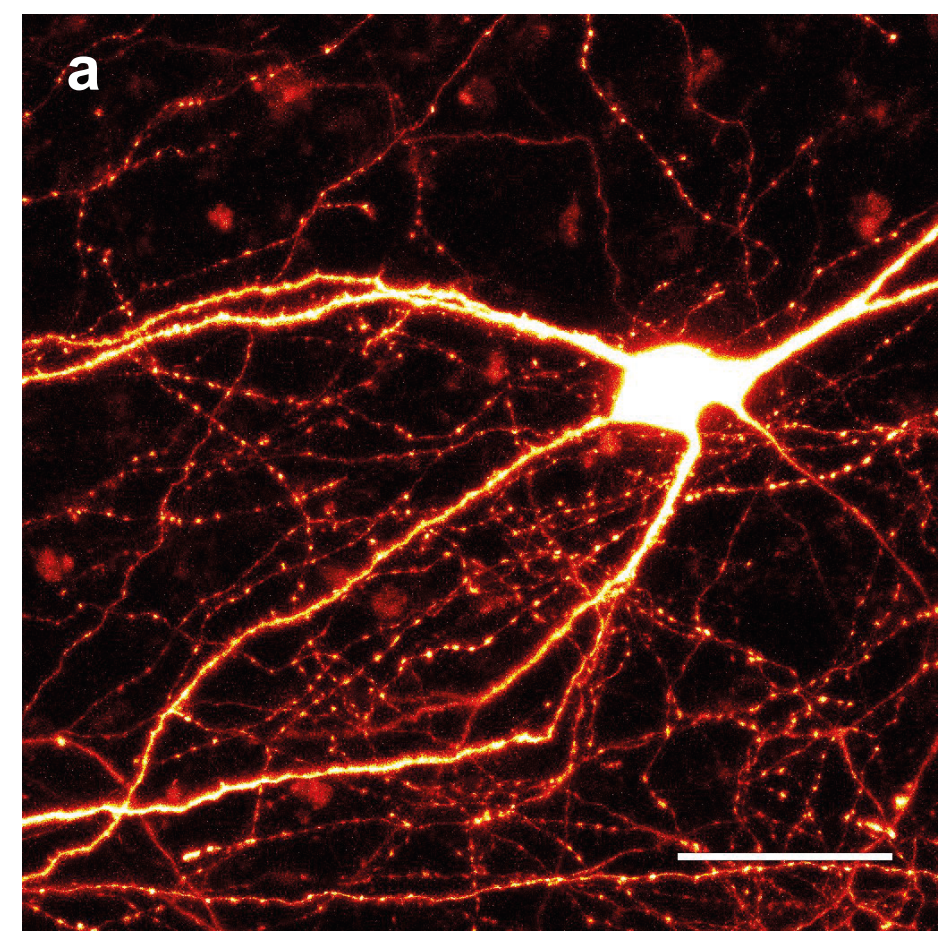

Click here to download Figure Figure 4 31072018.ai $\stackrel{\star}{ }$

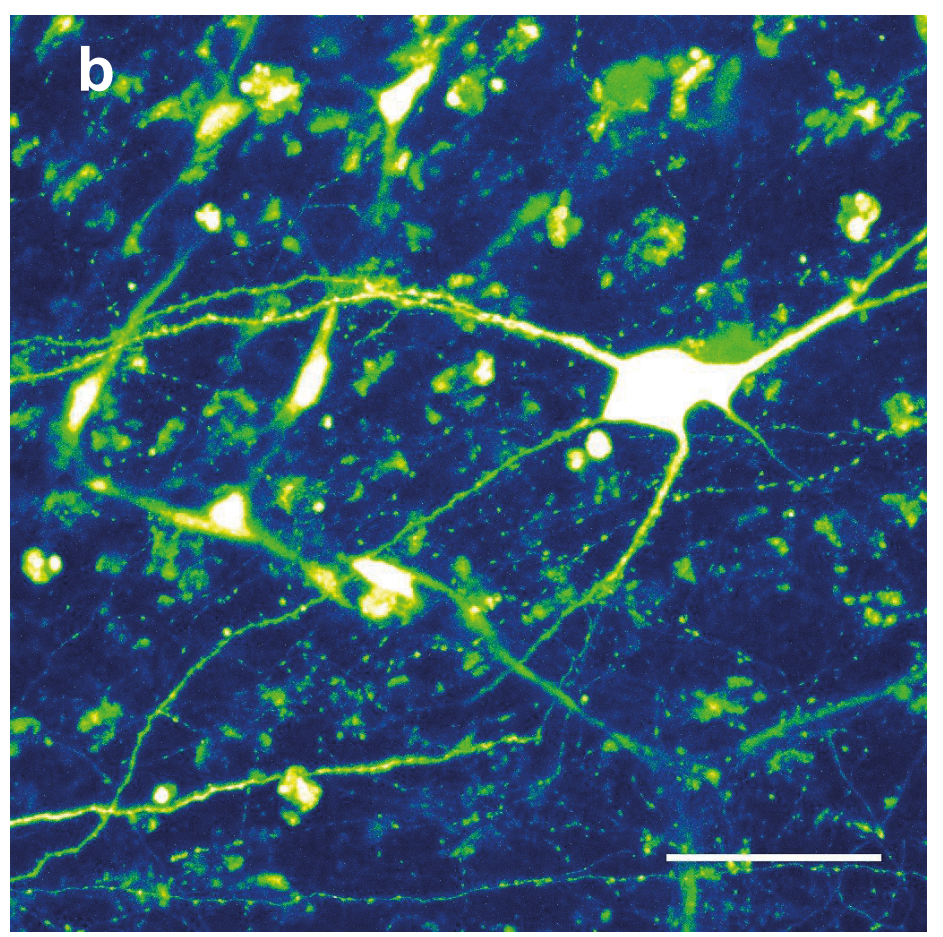



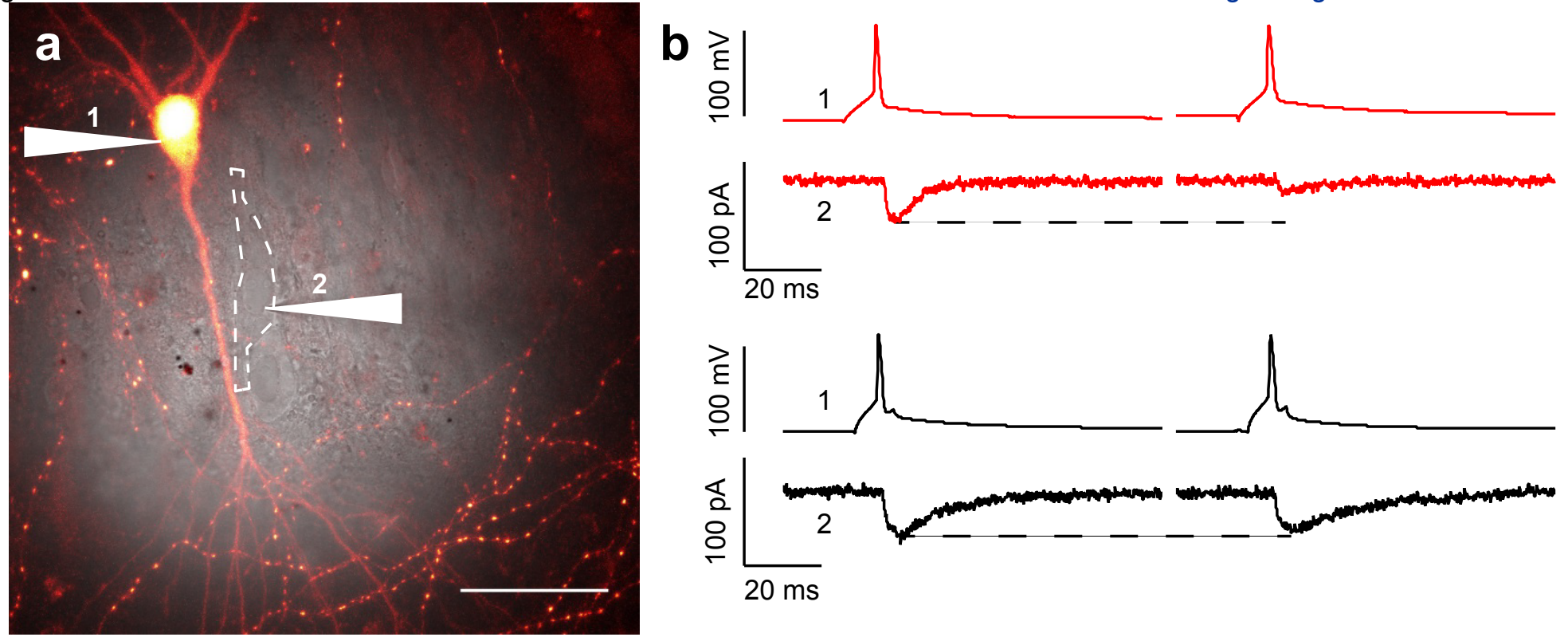

C
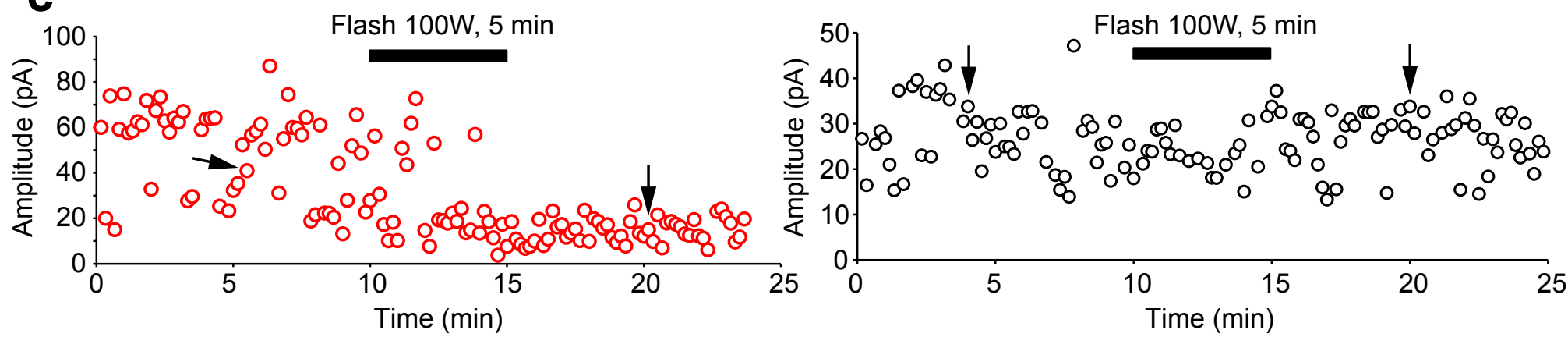

d
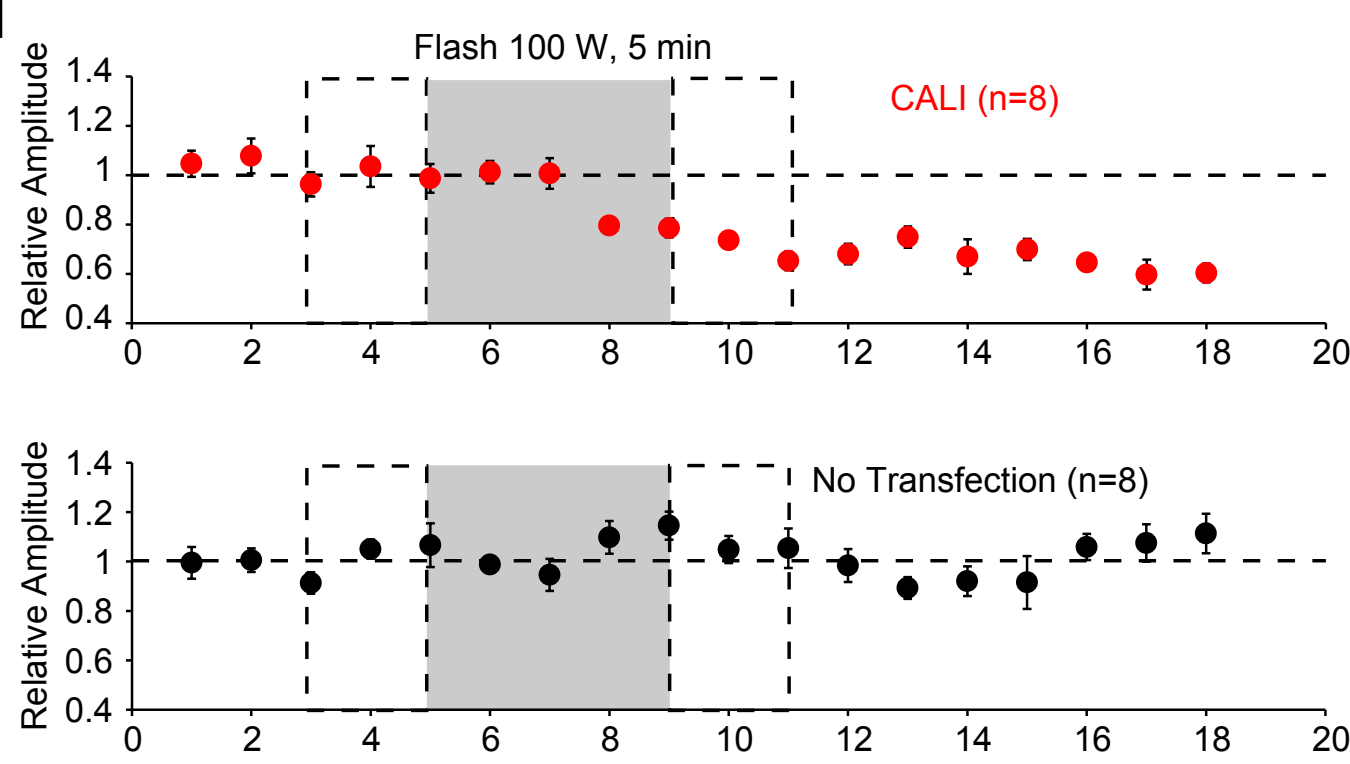

e

Comparison
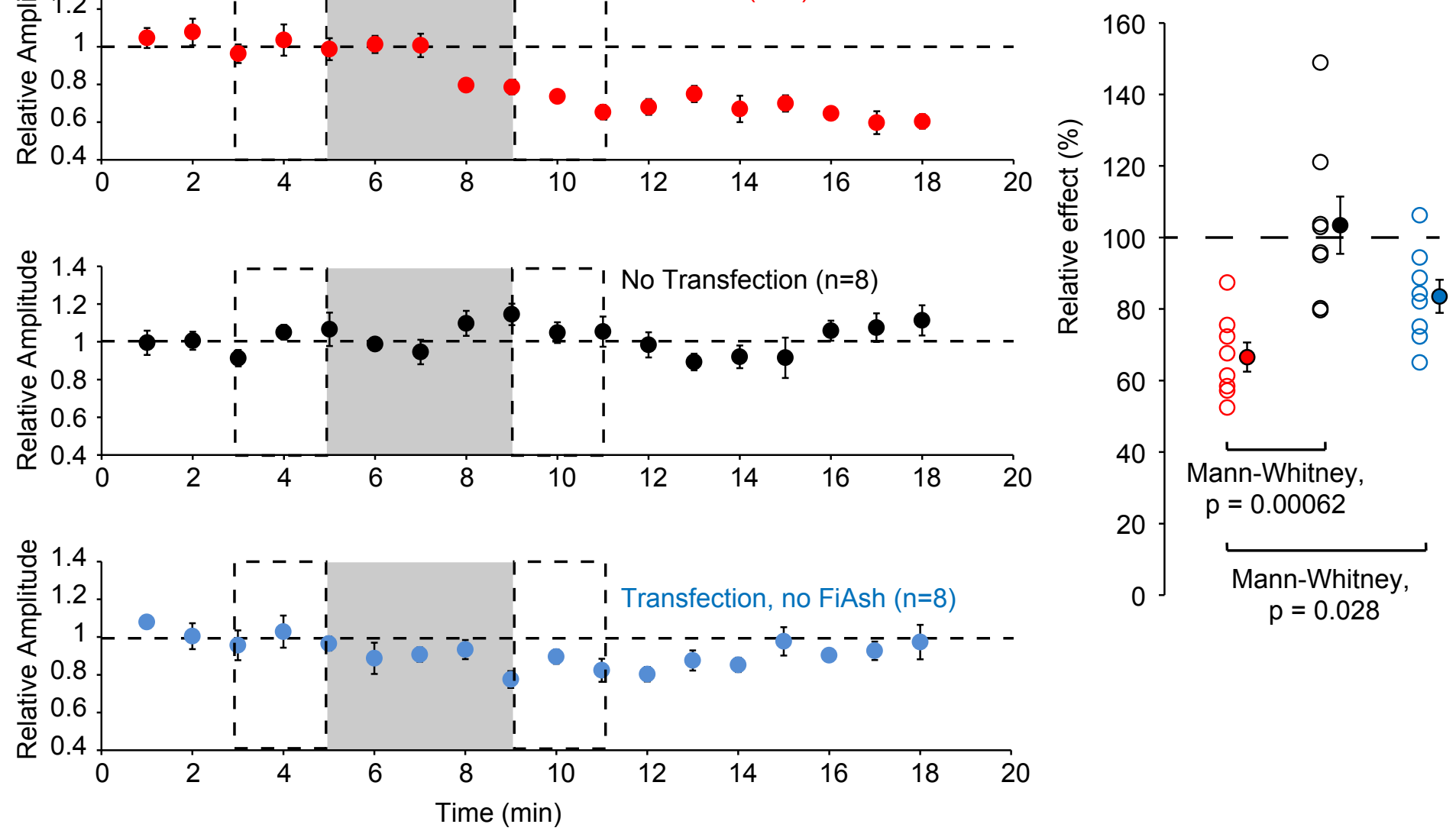

$p=0.028$ 
Table 1 : Mass Spectrometric identification of V0c tryptic peptides

\begin{tabular}{|c|c|c|c|c|c|c|}
\hline $\begin{array}{l}\text { Peptide } \\
\text { name }\end{array}$ & $\begin{array}{c}\text { Observed } \\
\mathrm{m} / \mathrm{z}\end{array}$ & $\begin{array}{c}\mathrm{Mr} \\
\text { (expected) }\end{array}$ & $\begin{array}{c}\mathrm{Mr} \\
\text { (calculated) }\end{array}$ & $\begin{array}{l}\text { Error } \\
\text { (ppm ) }\end{array}$ & Charge & Peptide sequence and modification \\
\hline \multirow{5}{*}{ PEP1 V0c } & 512.5554 & 1534.6443 & 1534.6439 & 0.23 & 3 & GSHMASMTGGQQMGR \\
\hline & 776.3265 & 1550.6384 & 1550.6388 & -0.27 & 2 & GSHMASMTGGQQMGR + Oxidation (M) \\
\hline & 517.8872 & 1550.6396 & 1550.6388 & 0.51 & 3 & GSHMASMTGGQQMGR + Oxidation (M) \\
\hline & 523.2186 & 1566.6341 & 1566.6337 & 0.20 & 3 & GSHMASMTGGQQMGR + 2 Oxidation (M) \\
\hline & 528.5501 & 1582.6283 & 1582.6287 & -0.21 & 3 & GSHMASMTGGQQMGR + 3 Oxidation (M) \\
\hline \multirow{4}{*}{ PEP2 V0c } & 1050.8084 & 3149.4032 & 3149.3933 & 3.16 & 3 & NNPEYSSFFGVMGASSAMVFSAMGAAYGTAK \\
\hline & 1056.1371 & 3165.3894 & 3165.3882 & 0.39 & 3 & NNPEYSSFFGVMGASSAMVFSAMGAAYGTAK + Oxidation (M) \\
\hline & 1061.4695 & 3181.3866 & 3181.3831 & 1.10 & 3 & NNPEYSSFFGVMGASSAMVFSAMGAAYGTAK + 2 Oxidation (M) \\
\hline & 1066.8012 & 3197.3816 & 3197.3780 & 1.13 & 3 & NNPEYSSFFGVMGASSAMVFSAMGAAYGTAK + 3 Oxidation (M) \\
\hline \multirow{8}{*}{ PEP3 V0c } & 946.4945 & 1890.9745 & 1890.9729 & 0.82 & 2 & SGTGIAAMSVMRPELIMK \\
\hline & 631.3309 & 1890.9710 & 1890.9729 & -1.03 & 3 & SGTGIAAMSVMRPELIMK \\
\hline & 636.6633 & 1906.9682 & 1906.9678 & 0.17 & 3 & SGTGIAAMSVMRPELIMK + Oxidation (M) \\
\hline & 954.4919 & 1906.9692 & 1906.9678 & 0.72 & 2 & SGTGIAAMSVMRPELIMK + Oxidation (M) \\
\hline & 641.9949 & 1922.9630 & 1922.9628 & 0.11 & 3 & SGTGIAAMSVMRPELIMK + 2 Oxidation (M) \\
\hline & 962.4891 & 1922.9637 & 1922.9628 & 0.51 & 2 & SGTGIAAMSVMRPELIMK + 2 Oxidation (M) \\
\hline & 970.4858 & 1938.9570 & 1938.9577 & -0.34 & 2 & SGTGIAAMSVMRPELIMK + 3 Oxidation (M) \\
\hline & 647.3277 & 1938.9613 & 1938.9577 & 1.86 & 3 & SGTGIAAMSVMRPELIMK + 3 Oxidation (M) \\
\hline \multirow{4}{*}{ PEP SLYD } & 798.3808 & 1594.7470 & 1594.7484 & -0.83 & 2 & DVFMGVDELQVGMR \\
\hline & 806.3791 & 1610.7437 & 1610.7433 & 0.29 & 2 & DVFMGVDELQVGMR + Oxidation (M) \\
\hline & 543.2528 & 1626.7366 & 1626.7382 & -0.99 & 3 & DVFMGVDELQVGMR + 2 Oxidation (M) \\
\hline & 814.3766 & 1626.7386 & 1626.7382 & 0.26 & 2 & DVFMGVDELQVGMR + 2 Oxidation (M) \\
\hline
\end{tabular}


Click here to access/download Supplementary Material NewSupplementary.pdf 